\title{
Inverse problem for partial differential equations in social networks
}

\author{
T. Zvonareva ${ }^{1 *}$, O. Krivorotko ${ }^{1,2}$, S. Kabanikhin ${ }^{1,2}$ \\ ${ }^{1}$ Novosibirsk State University, Novosibirsk, Russia \\ ${ }^{2}$ Institute of Computational Mathematics and Mathematical Geophysics SB RAS, Novosibirsk, Russia \\ *e-mail: zvonareva-tanyushka@mail.ru
}

Key words: inverse problems, partial differential equations, social processes, diffusive logistic model, optimization, gradient method, optimization, tensor train decomposition, regularization

Motivation and Aim: A lot of functions are available on the social network: access for informing relatives (Facebook's Safety Check service), publishing data about missing friends (the Google Person Finder service) or promptly informing users of an impending threat and their further actions in case of emergencies (Alerts from Twitter). Such processes can be described by the diffusive logistic mathematical model that characterizes information dissemination in social networks [1]. The type of information is determined by the coefficients of the mathematical model and the initial conditions of the problem. To control and predict the type of information in social networks it is necessary to refine the model coefficients and initial data by some additional measurements (the inverse problem) [2].

Methods and Algorithms: One way to solve the inverse problem for partial differential equations is to reduce it to an optimization problem, where the misfit function characterizes the quadratic deviation of the model data from the experimental one. To find the local minimum of misfit function the gradient methods are applied. In most cases, it is necessary to determine the global minimum of the objective function. A new computational method based on tensor train decomposition [3] is applied to solve the optimization problem. The idea of proposed method is to extract the tensor structure of the optimized functional and use it for optimization.

Results: The reconstructed coefficients and initial data of mathematical models of social networks (as an example it is considered Digg.com and Twitter [4]) allows one to refine the information dynamic and give the recommendation for control it.

Acknowledgements: Supported by the Ministry of Education and Science of Russian Federation, by the President Grant of Russian Federation (No. MK-1214.2017.1) and by the grant No. 18-71-10044 of Russian Scientific Found (RScF).

\section{References}

1. Dai G., Ma R., Wang H., Wang F., Xu K. (2015) Partial differential equations with robin boundary condition in online social networks. Discrete and Continuous Dynamical Systems Series B. 20(6):16091624.

2. Kabanikhin S.I. (2008) Definitions and examples of inverse and ill-posed problem. Journal of Inverse and Ill-Posed Problems. 16(4):317-357.

3. Zheltkova V.V., Zheltkov D.A., Grossman Z., Bocharov G.A., Tyrtyshnikov E.E. (2017) Tensor based approach to the numerical treatment of the parameter estimation problems in mathematical immunology. Journal of Inverse and Ill-posed Problems. 26(1):51-66.

4. Wang H., Wang F., Xu K. (2013) Modeling information diffusion in online social networks with partial differential equations. arXiv:1310.0505 [cs.SI]. 